\title{
MRI-based texture analysis: a potential technique to assess protectors against induced-liver fibrosis in rats
}

\author{
Doaa Mahmoud-Ghoneim¹, Amr Amin², Peter Corr ${ }^{3}$ \\ ${ }^{1}$ Physics Department, ${ }^{2}$ Biology Department, Faculty of Science; ${ }^{3}$ Radiology Department, \\ Faculty of Health and Medical Science, UAE University, United Arab Emirates
}

Background. In this study, the protective effect of extract of Moringa oleifera against carbon tetrachloride $\left(\mathrm{CCl}_{4}\right.$ )-induced liver fibrosis in rats was evaluated using Magnetic Resonance Imaging-Texture Analysis (MRI-TA) and the results were compared to liver function tests and histopathology.

Methods. Twenty-eight male Wistar rats were randomly divided into 4 groups: a) the normal control group (C) received an intra-gastric administration of vehicle for eight weeks; b) the fibrosis group (F) received an intra-peritoneal administration of $\mathrm{CCl}_{4}$ twice a week for eight weeks; c) the silymarin group (S) received 0.2 $\mathrm{g} / \mathrm{kg}$ orally once a day for eight weeks along with $\mathrm{CCl}_{4} ;$ d) the $\mathrm{M}$. oleifera protected group $(M)$, received an intra-gastric dose at $0.5 \mathrm{~g} / \mathrm{kg}$ for 8 weeks concomitantly with $\mathrm{CCl}_{4}$. Histopathology and liver function were performed and both confirmed protection against $\mathrm{CCl}_{4}$-induced liver fibrosis.

Results. The fibrosis index showed a remarkable increase in collagen-I contents in the $\mathrm{CCl}_{4}$-injured animals $(12.73 \pm 2.37 \%)$ while fibrotic indices were significantly less in liver tissues of Moringa-treated and silymarin-treated animals $(5.23 \pm 0.13 \%$ and $1.23 \pm 1.01 \%$, respectively). MRI-TA results were consistent with previous histopathological findings. Classification of MRI-TA parameters for the C, F, and $M$ groups showed that the F group was separated from both $M$ and $C$ groups on the MDF-1 axis (Most Discriminating Parameters-1) whereby this group always had negative values. The $C$ and $M$ groups clustered closely on the same axis with positive values. Very similar results were obtained from classification of the $C, F$ and $S$ groups. The texture parameters used in this study measure the coarseness of the imaged tissue, which is influenced by the collagen contents and distribution, that are known to be increased in fibrosis and inhibited by antifibrotic drugs thus affecting image classification.

Conclusions. Based on our findings, MRI-TA can be established as a potential tool for assessing the protective or therapeutic effects of tested antifibrotic drug/s. M. oleifera exhibits a partial hepatoprotective effect on rats treated with $\mathrm{CCl}_{4}$ which was proven by histopathology and liver function tests and indicated by MRI-TA performed on liver samples. We recommend MRI-TA as a potential tool for a simpler, easier, and faster way of indicating the therapeutic effect of antifibrotic drugs.

Key words: MRI; liver fibrosis; Moringa oleifera; texture analysis

Received 23 December 2008

Accepted 9 February 2009

Correspondence to: Prof. Amr Amin, Biology Department,

Faculty of Science, UAE University,Al-Ain, P.O. Box 17551, United Arab Emirates; Phone: +971-3-7134381; Fax: +971-3-7671291; E-mail: a.amin@uaeu.ac.ae 


\section{Introduction}

Hepatic fibrosis is a dynamic and complicated pathological condition that occurs in response to chronic hepatocellular injury. Liver fibrosis takes place as a result of many chronic liver diseases, such as hepatitis viral infection, including hepatitis $B$ and hepatitis C. It is characterized by altered hepatic function and an excessive accumulation of extracellular matrix proteins, including collagen, which occurs in most types of chronic liver diseases. The progression of liver fibrosis often develops into irreversible cirrhosis and is associated with liver cancer. ${ }^{1-3}$ It is therefore imperative to reduce the damage to the liver and to slow down the progress of liver injury into fibrosis and cancer. Oxidative stress may be a common factor in chronic liver diseases of different etiologies. ${ }^{4}$ Many agents have been proposed for the prevention and treatment of fibrosis. New clinical approaches need to be developed to improve the efficiency of current treatments, which require continuous trials on new drugs with a therapeutic or protective effect against live fibrosis. However, the gold standard diagnostic method of any therapeutic follow-up of liver damage remains biopsy analysis. Biopsy requires expensive and complicated biochemical and histopathological procedures that are irreproducible and unsuitable for large population screening. ${ }^{5} \mathrm{New}$ techniques, which are less expensive and more reproducible, are continuously being investigated to provide a reliable preliminary evaluation of antifibrotic effects of novel tested drugs. Magnetic Resonance Imaging (MRI) has been successful in evaluating liver condition by using different acquisition sequences. ${ }^{6,7}$ Automated image analysis methods, particularly texture analysis, when applied to different imaging modalities, are capable of providing quantitative data on a liver pathological condition, and therefore provide a powerful tool of automated characterization. ${ }^{8-10}$ Previous studies have also demonstrated that TA of MR images can characterize tissue condition during degeneration and regeneration processes, as well as in relation to collagen contents. ${ }^{11,12}$ MRI analysis is reproducible and can be rapidly applied. It can also be repeated many times over the same site without any hazardous or destructive effects to the tissue. Most importantly, MRI can be applied in-vivo and ex-vivo. ${ }^{11,12}$ Taken together, MRI-TA offers a promising tool for monitoring the histopathological status of liver tissues and throughout therapeutic or preventive testing against fibrosis.

MRI-TA is studied in this work as a new approach applied on liver in order to predict the protective effect of a local plant, Moringa oleifera, suggested to have an antifibrotic effect; and compare the results to those of silymarin as a standard treatment of fibrosis. Conventional histopathological and liver function tests were performed and compared to MRI-TA results. To our knowledge, MRITA has not been previously applied to assess the therapeutic effects of antifibrotic drugs on liver.

Various parts of the M. oleifera Lam tree have been studied for several pharmacological applications. The plant extract has been shown to possess antioxidant activity ${ }^{13}$, hypocholesterolemic, anti-inflammatory and antimicrobial effects. Moringa seeds are known as a rich source of oil, protein and many bioactive compounds. ${ }^{14-18}$ Seeds extracts have also been shown to be potent anti-microbial, antigenotoxic, anti-inflammatory and anti-tumour remedies. ${ }^{19-21}$ A recent study reported that Moringa seed oil had the strongest anti-fungal activity against a zoophilic dermatophyte that is known to cause severe inflammatory reactions in humans. ${ }^{22}$

The antioxidant potential of $M$. oleifera might allow it to succeed where other anti- 
oxidants have failed in preventing hepatic fibrosis. ${ }^{23}$ Taken together, these findings support the suggestion that $M$. oleifera is a promising herbal remedy for the treatment of hepatic fibrosis. Investigations are currently underway to unravel the biochemical basis of Moringa's protection against $\mathrm{CCl}_{4}$-induced liver fibrosis.

\section{Materials and methods}

\section{Plant extraction}

Mature seeds of $M$. oleifera were collected in Al-Ain city, U.A.E. The plant was authenticated and specimen identification numbers are kept in the Biology's herbarium for future reference. A hundred grams of dried Moringa oleifera were mixed in $1000 \mathrm{ml}$ of $70 \%$ ethanol. Every $10 \mathrm{gm}$ of ground $\mathrm{M}$. oleifera (Lam.) seeds were mixed with 100 $\mathrm{ml}$ of $70 \%$ ethanol in $250-\mathrm{ml}$ conical flask. The mixture was then irradiated by microwave for two minutes. ${ }^{24}$ The extracts were finally filtered through gauze and evaporated under vacuum at $40^{\circ} \mathrm{C}$ using a rotary evaporator. The dried extract was dissolved in distilled water before administration.

\section{Animals}

Adult Wistar male rats having with the weight ranging from 180-220 $\mathrm{g}$ were obtained from the Animal House, UAE University Al-Ain, U.A.E. They were maintained on a standard pellet diet and tap water ad libitum and were kept in polycarbonate cages with wood chip bedding under a $12 \mathrm{~h}$ light/dark cycle and room temperature of $22-24^{\circ} \mathrm{C}$. The rats were acclimatized to the environment for two week prior to experimental use. This study was conducted following the guidelines of the Animal Research Ethics Committee of U.A.E University.

\section{Experimental design}

The rats were randomly divided into four groups: a) the normal control group $(C$; $\mathrm{n}=7$ ) received an intra-gastric administration of vehicle only for eight weeks; b) the fibrosis group $(\mathrm{F} ; \mathrm{n}=7)$ received an intraperitoneal administration of $\mathrm{CCl}_{4}$ twice a week for eight weeks; c) the silymarin group $(\mathrm{S}, \mathrm{n}=7)$ received $0.2 \mathrm{~g} / \mathrm{kg}$ orally once a day for eight weeks along with $\mathrm{CCl}_{4} ; \mathrm{d}$ ) $M$. oleifera protected group $(M ; n=7)$, received an intra-gastric dose of $0.5 \mathrm{~g} / \mathrm{kg}$ for 8 weeks concomitantly with $\mathrm{CCl}_{4}$. At the end of eight weeks, one day after the last administration and after induction of ether anesthesia, blood samples were drawn from the eye retro-orbital plexus from all the rats prior to the excision of organ tissues. The serum was stored at $-80^{\circ} \mathrm{C}$ after separation until it was assayed as described below. Liver was excised and specimens were placed in plastic containers with $10 \%$ buffered formaldehyde solution and kept overnight for following MRI.

\section{Detection of serum markers}

The activities of serum aspartate aminotransferase (AST) and alanine aminotransferase (ALT) were assayed by spectrophotometric analysis (Bio-Merieux, RCS Lyon, France) according to the manufacturer's instructions.

\section{Histochemistry}

Liver tissue samples from all animals were processed for light microscopy and embedded in paraffin blocks, which were cut to obtain $5 \mu \mathrm{m}$ thick sections and were mounted on slides. Sections were immunostained with anti-collagen I antibodies (Santa Cruz, CA, USA). Sections were examined using a Leica DMRB/E light microscope (Heerbrugg, Switzerland). 

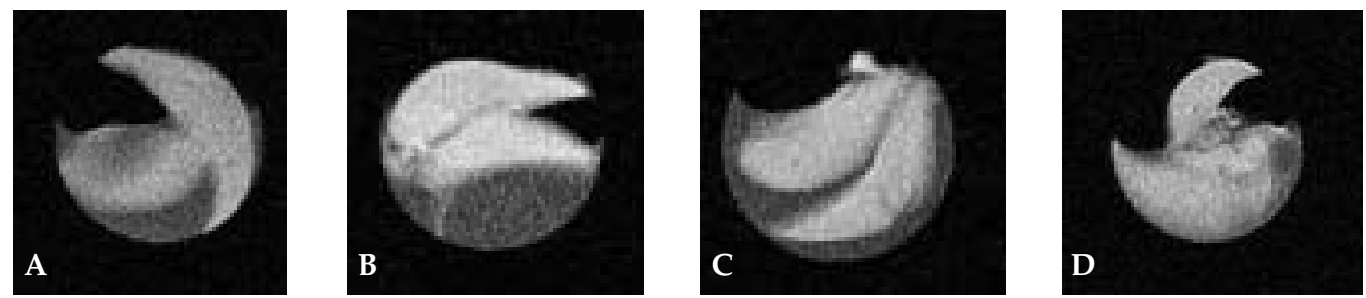

Figure 1. MRI image of liver specimens from: a) control, b) fibrotic, c) Moringa-treated and d) silymarin-treated rat groups. Images were acquired on the excised liver samples using the 1.5-T MRI system and T1-weighted Spin Echo sequence.

\section{Statistical Analysis}

SPSS (version 10) (SPSS Inc., Chicago, IL, USA) was used to carry out a one-way analysis of variance (ANOVA) on the histochemistry results. When significant differences were detected by ANOVA, analyses of differences between the means of the treated and control groups were performed using Dunnett's t-test.

\section{Fibrosis index calculation}

Microscopic images of 100x magnification were acquired for collage-I immunostained liver slides giving a resolution of $2.6 \mu \mathrm{m}^{2}$. Images were initially encoded on 24-bits per pixel three channels (Red, Green, and Blue). The percentage of fibrosis in the liver tissue was determined using a computer assisted automated image analysis program made by the authors using Matlab ${ }^{\odot}$ (version 7.00, (c) 1984-2004, The MathWorks Inc). It gives the fibrosis index by calculating the ratio of type I collagen fibers (immunostained areas) to the whole liver area on a slide. ${ }^{25,26}$ Histopathological evaluation was performed in four different sites per section for each treatment $(n=7)$. The average value was then calculated for each experimental group to give the fibrosis index for each group.

\section{$M R I$ and regions of interest}

The specimens were imaged on a 1.5-T MR scanner (General Electric Medical Systems,
Milwaukee). The plastic containers containing liver specimens were attached on a standard MR phantom. Specimens were imaged within a standard quadrature head coil. A Spin Echo T1-weighted sequence was used to image all specimens $(\mathrm{TE}=14 \mathrm{~ms}$, $\mathrm{TR}=500 \mathrm{~ms}$, flip angle $=90^{\circ}$ ), with a field of view of $12 \mathrm{~cm}$, matrix size $256 \times 256$, slice thickness of $5 \mathrm{~mm}$, and NEX=1. DICOM images were converted to 8 bits-per-pixel bitmap images, and one region of interest (ROI), covering the largest possible homogenous area of liver was selected for texture analysis (about 250 pixels) (Figure 1).

\section{Texture analysis and image processing}

Microscopic images were converted to greylevels bitmap images suitable for texture analysis, and ROIs of about 30000 pixels were selected on each image.-

Texture analysis of microscopic and MR images was performed using Runlength Matrix and Gradient Matrix methods. ${ }^{27,28}$ These two matrices were chosen since they are sensitive to texture coarseness and can therefore characterize collagen accumulation. ${ }^{12}$ Collagen accumulations produce a coarse image texture, while the absence of collagen gives fine texture, which is used as a base for discrimination between groups. The Runlength Matrix method was proposed by Galloway. ${ }^{27}$ It calculates the number of runs $p(i, j)$ of greylevel $i$ and length $d$ in the direction of angle $\theta$ from one or more of the 
Table 1. Effect of Moringa oleifera (M) and silymarin (S) on serum markers of liver damage in $\mathrm{CCL}_{4}$-induced fibrotic $(\mathrm{F})$ and control $(\mathrm{C})$ groups. Values are expressed as mean $\pm \mathrm{SEM}(\mathrm{n}=7) .{ }^{* *} \mathrm{P}<0.001$ vs. control group; **P $<0.001$ vs. fibrotic group.

\begin{tabular}{ccccc}
\hline & $\begin{array}{c}\text { C } \\
(\mathrm{n}=7)\end{array}$ & $\begin{array}{c}\mathbf{F} \\
(\mathrm{n}=7)\end{array}$ & $\begin{array}{c}\mathbf{M} \\
(\mathrm{n}=7)\end{array}$ & $\begin{array}{c}\text { S } \\
(\mathrm{n}=7)\end{array}$ \\
\hline $\begin{array}{c}\mathrm{AST} \\
(\mathrm{IU} / \mathrm{L})\end{array}$ & $43.68 \pm 3.53^{* * *}$ & $112.60 \pm 25.90^{* * *}$ & $58.22 \pm 10.26^{* * *}$ & $65.83 \pm 7.66^{* * *}$ \\
$\begin{array}{c}\text { ALT } \\
(\mathrm{IU} / \mathrm{L})\end{array}$ & $46.71 \pm 1.86^{* * *}$ & $128.88 \pm 19.74^{* * *}$ & $60.46 \pm 8.55^{* * *}$ & $71.98 \pm 9.73^{* * *}$ \\
\hline
\end{tabular}

values $\left(0^{\circ}, 45^{\circ}, 90^{\circ}, 135^{\circ}\right)$. In coarse textures, relatively long greylevel runs would be more common, while fine textures should primarily comprise short runs. Some of the parameters extracted from this method are: longrun emphasis, short-run emphasis, runlength nonuniformity, and greylevel nonuniformity. The Gradient Matrix (GrM) characterises the distribution of greylevel differences around a pixel $g(x, y)$, located at row $x$ and column $y$, within a "mask" of neighbouring pixels (usually $3 \times 3$ or $5 \times 5$ ). The mask is applied pixel by pixel over the whole image or ROI. ${ }^{28}$ Some of the GrM parameters are: mean, variance, skewness and kurtosis.

\section{Features selection and classification}

Features selection aims to identify the most discriminating parameters from each matrix that separate the different classes most efficiently. The Fisher-coefficient (F-coefficient) was calculated for this purpose, giving the ratio of between class variance to within class variance for each parameter. The ten parameters of the highest F-coefficient were entered into Linear Discriminant Analysis (LDA) for classification. LDA aims to find a linear transformation matrix so that the ratio of the within-class scatter matrix to between-class scatter matrix is maximized. Such a transformation is composed of eigenvectors corresponding to the largest eigenvalues of this ratio of matrices; more details about the classification method can be found elsewhere. ${ }^{29}$ The classification results of LDA are represented graphically as the relationship between the most discriminating features (MDF). The number of MDF axes is less than the number of the output classes by one. In this study, we have three output classes for each classification attempt, thus classes would be represented on two axes, MDF1 and MDF2. Texture analysis, features selection, and classification were performed using MaZda-B11 software (version 4.5, (c)1999-2006 by Piotr Szczypinski) which were initially developed under the auspices of the COST action B11 European project. More details about the software can be found in. ${ }^{30}$

\section{Results}

\section{Effect of M. oleifera on liver function}

The activity of serums AST and ALT are useful indicators of the extent of liver injury following $\mathrm{CCl}_{4}$ treatment. Table 1 shows AST and ALT activities at the end of the experiment. Increases of 2.6- and 2.8-fold in the activity of AST and ALT, respectively, were observed in the $\mathrm{CCl}_{4}$ group compared to the control group $(p<0.001)$. Treatment with Moringa decreased the $\mathrm{CCl}_{4}$-induced levels of AST and ALT by $47 \%$ and $52 \%$ $(p<0.001)$, respectively. Similarly, treatment with silymarin in $\mathrm{CCl}_{4}$ treated rats reduced the AST level by $40 \%(p<0.001)$. It is worth mentioning here that both biochemical and histopathological results confirmed that Moringa alone did not have any toxic effects on rat liver tissues (data not shown). 

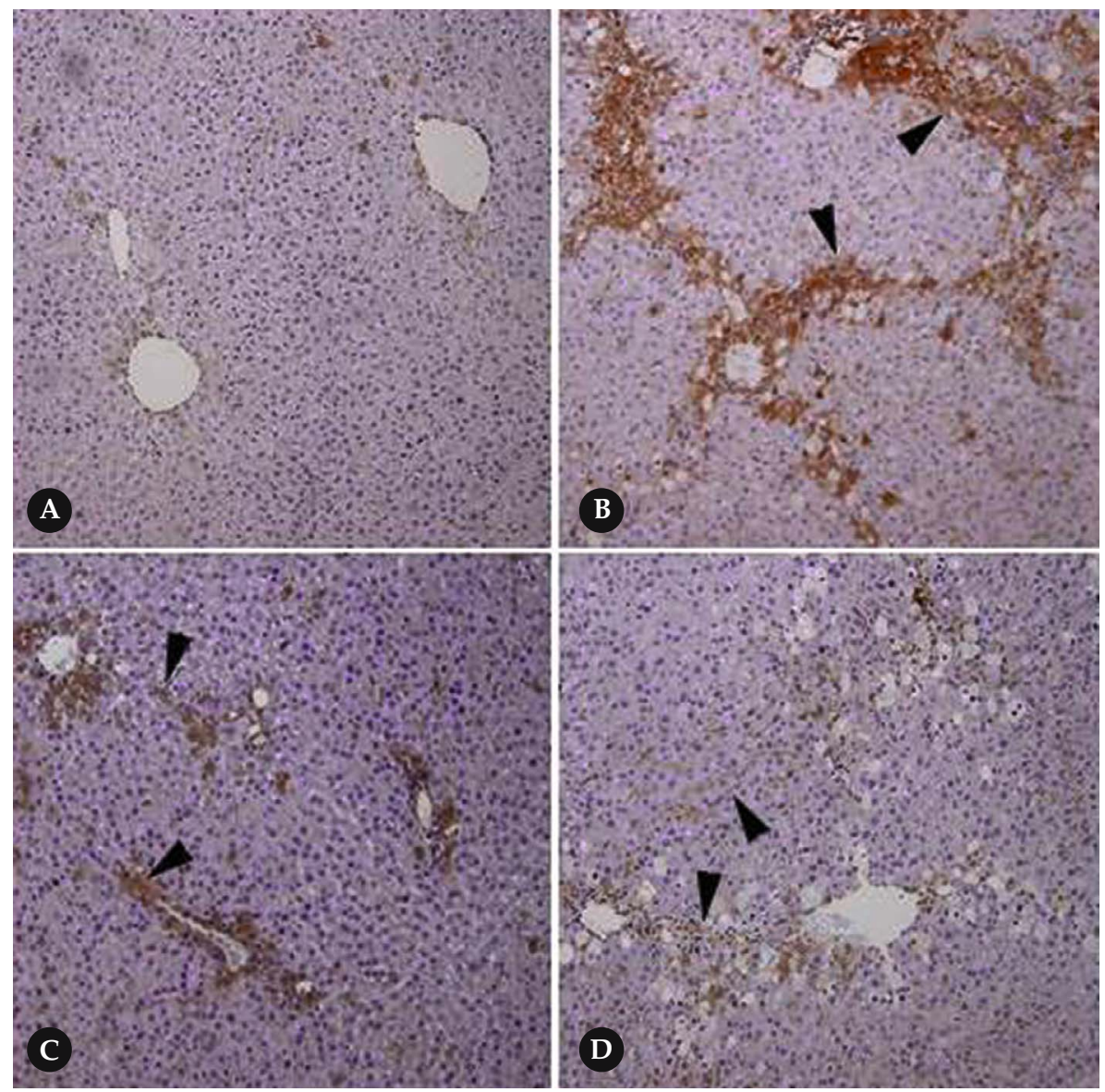

Figure 2. Histological sections of liver showing Collagen-I. A) control, B) fibrotic, C) Moringa, and D) silymarin. (A) Section of liver tissue of control rat showing normal architecture with central veins and radiating cords of hepatocytes. (B) Section of liver tissue of rats treated with $\mathrm{CCl}_{4}$ showing macrovesicular fatty change around central vein and large areas of necrosis fatty degeneration, necrosis, infiltration of inflammatory cells and apparent formation of fibrotic septa. (C) Section of liver tissue of rats treated with Moringa oleifera extract $(0.5 \mathrm{~g} / \mathrm{kg} \mathrm{b.wt})$ and $\mathrm{CCl}_{4}$ showing the presence of normal hepatic cords, absence

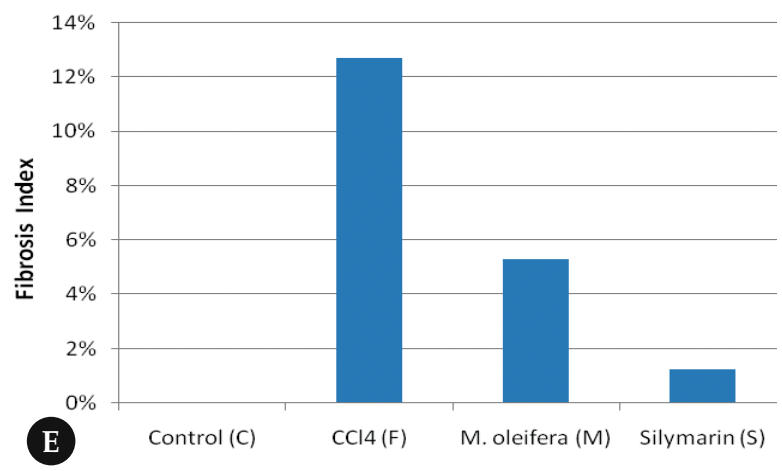
of necrosis and macrovesicular fatty change.

The degree of liver damage and fibrosis were significantly reduced in the M. oleifera $(0.5 \mathrm{~g} / \mathrm{kg} \mathrm{b}$. wt.) treated rats. (D) Section of liver tissue of rats treated with silymarin $\left(0.2 \mathrm{~g} / \mathrm{kg}\right.$ b.wt.) and $\mathrm{CCl}_{4}$ showing normal hepatic architecture, which was nearly similar to that of the healthy control. Collagen deposition (arrowheads) is indicated as brown-stained fibers in panel B-D. All sections were immunostained with anti-collagen I antibody, 100×. (E) Histogram representing the calculated fibrotic indices for all groups. The fibrosis index was calculated as the ratio between the connective tissue (immunostained area) and the whole liver area in the slide. 


\section{Pathological histology}

Collagen-I immunostaining of liver specimen examination showed no histological abnormalities and the absence of collagen strands in the extracellular hepatic matrix in normal control liver (Figure 2a). Liver tissue in $\mathrm{CCl}_{4}$-injured rats presented a gross nodular appearance as well as steatosis, cell necrosis and inflammatory infiltration compared to control rats. Histological abnormality in fibrotic model rat was characterized by the deposit of bundles of collagen in pericentral and midzonal areas of liver tissues (Figure 2b), which gave a fibrosis index of $12.73 \pm 2.37 \%$ (Figure 2e). Co-administration of $M$. oleifera $(0.5 \mathrm{~g} / \mathrm{kg})$ or silymarin $(0.2 \mathrm{~g} / \mathrm{kg})$ markedly alleviated the degree of liver fibrosis and significantly lowered collagen deposited (Figure 2c, d). Treatment with $M$. oleifera decreased in the fibrosis index by $5.23 \pm 0.13 \%$, while reduction with silymarin was by $1.23 \pm 1.01 \%$ (Figure 2e).

\section{Texture analysis and classification}

Texture analysis of microscopic images followed by LDA was always able to cluster samples belonging to the $F$ group as a homogenous class separate and distant from other classes (Figure 3a,b) and always separable on the MDF1 axis with positive values. On the other hand, class $\mathrm{S}$ tended to cluster with class C (Figure 3a); as did class $M$ (Figure $3 b$ ). Classes $C, S$ and $M$ always had negative values on MDF1 (Figures $3 a, b)$. MRI-TA classification results were similar to those of microscopic images. F was always classified as a separate class distant from other classes (Figure 4), while $\mathrm{M}$ and $\mathrm{S}$ classes clustered close to or even mixed with class C (Figure 4a,b). Class F was separable on MDF1, on which it had always negative values, unlike the classes $C$, $\mathrm{S}$ or $\mathrm{M}$, which that had positive values on the same axis. In all classification attempts, class $\mathrm{F}$ (true positive) did not show any classification errors with other classes and no sample that belonged to other classes (true negative) was classified as F. This gives a sensitivity and specificity of $100 \%$ for that test. This result in particular highlights the uniqueness of textural features of fibrotic liver tissue. In contrast, $\mathrm{C}, \mathrm{M}$ and $\mathrm{S}$ groups were occasionally misclassified with each other (Figures 3,4), indicating the presence of similar textural features.

\section{Discussion}

This study measured the consistency of results among MRI-TA, TA on microscopic images, histopathology and biochemical analysis, of the effect of crude extract of M. oleifera seeds on $\mathrm{CCl}_{4}$-induced liver fibrosis.

$\mathrm{CCl}_{4}$ is a well-known hepatotoxin. ${ }^{31,32}$ Continuous exposure to this toxin produces progressive liver injury and fibrosis, eventually causing cirrhosis, portal hypertension and death. ${ }^{33,34}$ The main cause of acute liver injury by $\mathrm{CCl}_{4}$ is free radicals of its metabolites. Activation of $\mathrm{CCl}_{4}$ by liver cytochrome P-450 generates methyltrichloride radicals $\left(\mathrm{CCl}_{3}\right)$. These radicals cause lipid peroxidation, which produces hepatocellular damage and enhanced production of fibrotic tissue. ${ }^{35}$ The results of this study demonstrate that treatment of rats with $M$. oleifera had a markedly protective effect against $\mathrm{CCl}_{4}$-induced hepatotoxicity in rats, as evidenced by decreased serum AST and ALT activities. Similarly, total flavonoids of Bidens pilosa L. has been shown to decrease the elevated level of ALT and AST in a $\mathrm{CCl}_{4}$-induced liver fibrosis model. ${ }^{36}$ It was evident from the histopatholgy and biochemical analysis that co-administration of M. oleifera seed extract with $\mathrm{CCl}_{4}$ provided significant protection against liver fibrosis. 

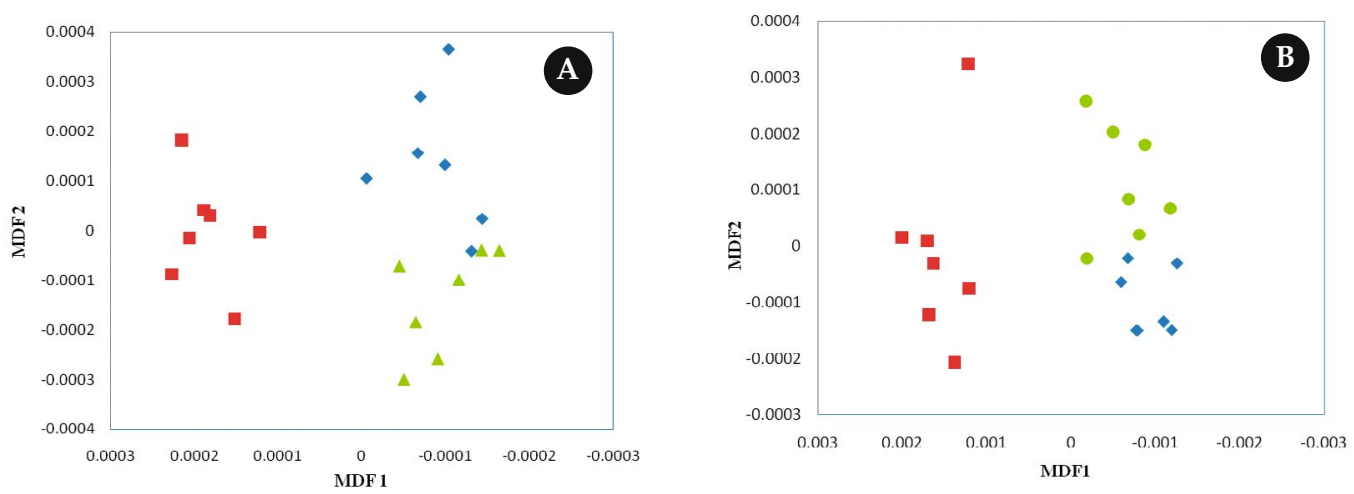

Figure 3. Linear Discriminant Analysis (LDA) of microscopic image texture showing: a) control class (C) ( $\downarrow)$, fibrotic class $(\mathrm{F})(\mathbf{\square})$, against silymarin class $(\mathrm{S})(\mathbf{\Delta})$. b) control class $(\mathrm{C})(\bullet)$, fibrotic class $(\mathrm{F})(\mathbf{\square})$, against Moringa class $(\mathrm{M})(\bullet)$. MDF1 and MDF2 are the most discriminating features axes that are used in LDA to represent the classification graphically.

Immunostaining of liver specimens showed a remarkable difference in collagen-I contents, as a fibrosis index (FI), in the experimental groups (Figure 2e). Control rats showed an absence of collagen strands in the extracellular hepatic matrix (Figure 2a) and this was set as zero FI. The fibrotic group showed a clear formation of collagen networks in the liver (Figure 2b); and therefore gave the highest FI (Figure 2e). The Moringa treated group, as well as silymarin-treated group, showed much less collagen accumulation (Figure $2 \mathrm{c}, \mathrm{d}$ ) and accordingly a lower FI (Figure 2e).

MRI-TA reveals textural changes related to minor or major pathological modifications that take place in tissue. These textural changes are basically alterations in the physical-chemical properties of the underlying tissue that affect the relaxation times (T1 and T2) in MRI, and, consequently, appear in the image as alterations in greylevel intensities (taking the healthy tissue as reference). These altered intensities are impercentible to the human eye and can only be measured using a texture analysis method. In this respect, texture analysis followed by automatic classification provides a powerful quantification tool of liver pathology, which offers a wide range of applications.
One of these fields of application is antifibrotic pharmaceutics research, which requires recurring essays. Collagen is particularly influential on texture. It has been demonstrated in the literature that collagen contents and distribution have a direct effect on texture discrimination on MR images. ${ }^{12}$ This is due to the collagen extracellular network, which gives a coarse texture for thicker networks and fine texture for thinner ones. ${ }^{12}$ Coarse texture gives longer runs using the Runlength method of texture analysis ${ }^{27}$; the Runlength matrix therefore seems to be a suitable method of texture analysis of fibrotic liver. LDA on both MRI- and microscopic image TA, showed that class $\mathrm{S}$ always clustered closely or even mixed with class $C$. This indicates that the $S$ experimental group has textural properties, which are themselves dependent on tissue composition, close to those of $\mathrm{C}$ group. This was confirmed by liver function tests (ALT and AST) and collagen-I contents indicated as FI. Similarly, the clustering of group $\mathrm{M}$ in close proximity to group $\mathrm{C}$ can be attributed to the closely related textural properties of those groups, which may also be related to their collagen contents.

MRI-TA correlated well with fibrosis histopathological results and showed a clear 

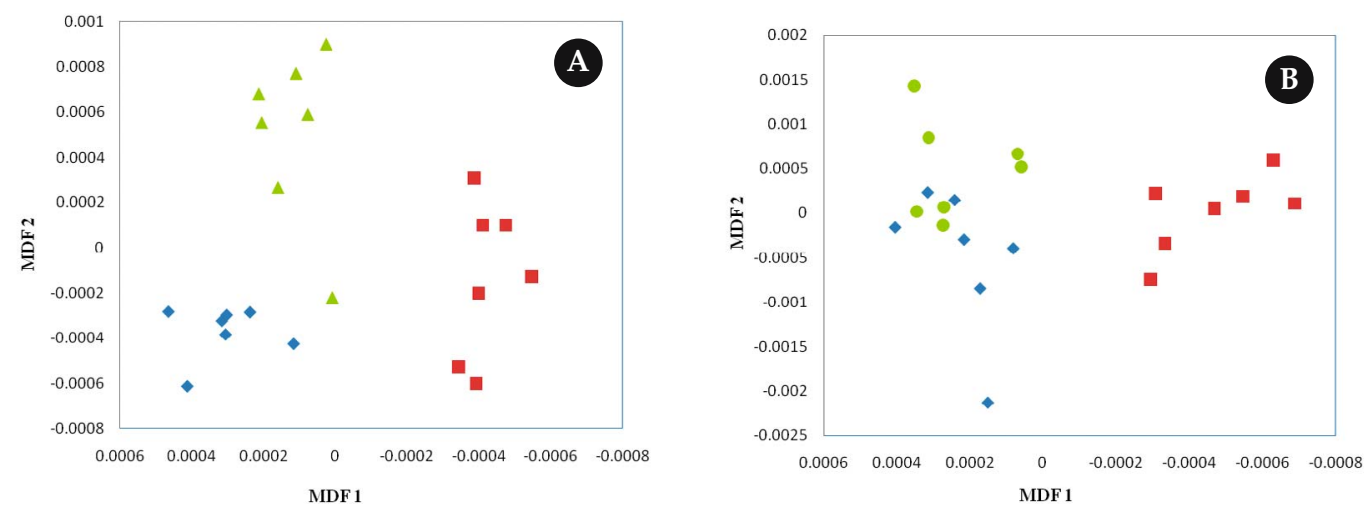

Figure 4. Linear Discriminant Analysis (LDA) of MRI-TA (Texture Analysis on MRI) showing: a) control class (C) $(\bullet)$, fibrotic class $(\mathrm{F})(\mathbf{\bullet})$, against silymarin class $(\mathrm{S})(\mathbf{\Delta})$. b) control class $(\mathrm{C})(\boldsymbol{\bullet})$, fibrotic class $(\mathrm{F})(\mathbf{\square})$, against Moringa class $(\mathrm{M})(\bullet)$. MDF1 and MDF2 are the most discriminating features axes that are used in LDA to represents classification graphically.

discrimination between group $\mathrm{F}$ on the one hand and other (C, M and S) groups on the other, with $100 \%$ sensitivity and specificity. MRI-TA results were also consistent with texture analysis of microscopic images, which suggests that the former method can be used as a reliable evaluation tool of liver condition during different treatments. Therefore, using an appropriate standard drug as a positive control, and with minimal biochemical and/or histopathological diagnostic procedures, the MRI-TA protocol can now provide a reliable diagnostic and monitory tool for testing new antifibrotic drugs. Further investigations are currently underway to identify and characterize Moringa's bioactive ingredients and to gain more molecular insights into its potent antifibrotic property.

\section{Conclusions}

MRI-based texture analysis provides a reliable alternative tool for primary evaluation of therapeutic effects. This technique would open a new door for drug assessment, since it is a rapid, less expensive and nondestructive tool. Moringa oleifera has shown a protective effect against liver fibrosis. This protection was confirmed by liver function tests and histopathological analysis and found to be measurable using MRI-TA.

\section{Acknowledgements}

This work was funded by Research Affairs at UAE University, UAE (Grant \# 01-042-11/07). The authors are grateful for Mr. Alaa Hamza for his technical assistance and Mr. Stuart Perry for image processing. The authors would also like to thank Mr. P.C. Mathewkutty, Mr. Solomon Raju Pothurajy and the entire technician team at Al-Ain Hospital, for their assistance in image acquisition.

\section{References}

1. Zou YH, Yang $\mathrm{Y}, \mathrm{Li} \mathrm{J}, \mathrm{Wu} \mathrm{Q}, \mathrm{Li} \mathrm{WP}, \mathrm{Lu} \mathrm{JT}$, et al. Potential therapeutic effects of a traditional Chinese formulation, BJ-JN, on liver fibrosis induced by carbon tetrachloride in rats. $J$ Ethnopharmacol 2008; 120: 452-7.

2. Lamireau A, Desmouliere P, Bioulac-Sage, Rosenbaum J. Mechanisms of hepatic fibrogenesis. Arch Pediatrie 2002; 9: 392-405. 
3. Okazaki I, Watanabe T, Inagaki Y. Recent advance in understanding mechanisms of fibrogenesis and fibrolysis in hepatic fibrosis. Nippon Shokakibyo Gakkai Zasshi. Jpn J Gastroenterol 2002; 99: 353-64.

4. Parola M, Robino G. Oxidative stress-related molecules and liver fibrosis. J Hepatol 2001; 35: 297306.

5. Bedossa P, Carrat F. Liverbiopsy: the best, not the gold standard. J Hepatol 2009; 50: 1-3.

6. Lewin M, Poujol-Robert A, Boëlle PY, Wendum D, Lasnier E, Viallon M, et al. Diffusion-weighted magnetic resonance imaging for the assessment of fibrosis in chronic hepatitis C. Hepatology 2007; 46: 658-65.

7. Bobekamp S, Kamel I, Solga S, Clark J. Can imaging modalities diagnose and stage hepatic fibrosis and cirrhosis accurately? J Hepatol 2009; 50: 1735.

8. Jirák D, Dezortová M, Taimr P, Hájek M. Texture analysis of human liver. J Magn Reson Imaging 2002; 15: 68-74.

9. Zhang X, Fujita H, Kanematsu M, Zhou X, Hara $\mathrm{T}$, Kato $\mathrm{H}$, et al. Improving the classification of cirrhotic liver by using texture features. Conf Proc IEEE Eng Med Biol Soc 2005; 1: 867-70.

10. Ganeshan B, Miles KA, Young RCD, Chatwin CR. Texture analysis in non-contrast enhanced CT: Impact of malignancy on texture in apparently disease- free areas of the liver. Eur J Radiol 2008; In press. [Epub ahead of print]

11. Mahmoud-Ghoneim D, Cherel Y, Lemaire L, de Certaines JD, Maniere A. Texture analysis of Magnetic Resonance Images of rats muscles during atrophy and regeneration. Magn Reson Imaging 2006; 24: 167-71.

12. Mahmoud-Ghoneim D, Bonny JM, Renou JP, de Certaines JD. Ex-vivo Magnetic Resonance Imaging Texture Analysis can discriminate genotypic origin in bovine meat. J Sci Food Agr 2005; 85: 629-32.

13. Arabshahi-D S, Devi V, Urooj A. Evaluation of antioxidant activity of some plant extracts and their heat, $\mathrm{pH}$ and storage stability. Food Chem 2007; 100: 100-5.

14. Ndabigengesere A, Narasiah KS. Quality of water treated by coagulation using Moringa oleifera seeds. Water Res 1998; 32: 781-91.

15. Ndabigengesere A, Narasiah KS, Talbot BG. Active agents and mechanism of coagulation of turbid waters using Moringa oleifera. Water Res 1995; 29: 703-10.
16. Muyibi SA, Evison LM. Moringa oleifera seeds for softening hardwater, Water Res 1995; 29: 1099105.

17. Gassenschmidt U, Jany KD, Tauscher B, Niebergall $\mathrm{H}$. Isolation and characterization of a flocculating protein from Moringa oleifera Lam. Biochim Biophys Acta 1995; 1243: 477-81.

18. Guevara AP, Vargas C, Sakurai H, Fujiwara Y, Hashimoto K, Maoka T, et al. An antitumor promoter from Moringa oleifera Lam. Mutation Res 1999; 440: 181-8.

19. Dayrit F, Alcantara A, Villasenor I. The antibiotic compound and its deactivation in aqueous solutions. Phil J Sci 1990; 119: 23-6.

20. Villasenor I. Bioactive metabolites from Moringa oleifera Lam. Kimika 1994; 10: 47-52.

21. Makkar HPS, Becker K. Nutrient and antiquality factors in different morphological parts of Moringa oleifera tree. J Agr Sci 1997; 128: 311-22.

22. Chuang P-H, Lee C-W, Chou J.-Y, Murugan M, Shieh B.-J, Chen H-M. Anti-fungal activity of crude extracts and essential oil of Moringa oleifera Lam. Bioresource Technol 2007; 98: 232-6.

23. Rice-Evans C. Implications of the mechanisms of action of tea polyphenols as antioxidants in vitro for chemoprevention in humans. Proc Soc Exp Biol Med 1999; 220: 262-6.

24. Pan X, Niu G, Liu H. Microwave-assisted extraction of tanshinones from Salvia miltiorrhiza bunge with analysis by high-performance liquid chromatography. J Chromatog 2001; 922: 371-5.

25. Salgado S, García J, Vera J, Siller F, Bueno M, Miranda A, et al. Liver cirrhosis is reverted by urokinase-type plasminogen activator gene therapy. Mol Ther 2000; 2: 545-51.

26. Salazar-Montes A, Ruiz-Corro L, López-Reyes A, Castrejón-Gómez E, Armendáriz-Borunda J. Potent antioxidant role of Pirfenidone in experimental cirrhosis. Eur J Pharmacol 2008; 595: 69-77.

27. Galloway MM. Texture analysis using greylevel run lengths. Comp Graph Image Proc 1975; 4: 1729.

28. Lerski RA, Straughan K, Schad LR, Boyce D, Bluml S, Zuna I. Magn Reson Imaging 1993; 11: 873-87.

29. Swets W. Using discriminant eigenfeatures for image retrieval. IEEE PAMI 1996; 18: 831-6. 
30. Materka A. MaZda and B11 User's Manual (19992002. (Download software and manual: http:// www.eletel.p.lodz.pl/merchant/mazda/order1_ en.epl)

31. Yu C, Wang F, Jin CL, Wu X, Chan WK, McKeehan WL. Increased carbon tetrachloride-induced liver injury and fibrosis in FGFR4-deficient nice. Am J Pathol 2002; 161: 2003-10.

32. Marucci L, Alpini G, Glaser SS, Alvaro D, Benedetti A, Francis $\mathrm{H}$, et al. Taurocholate feeding prevents $\mathrm{CCl}_{4}$-induced damage of large cholangiocytes through PI3-kinase-dependent mechanism, Am J Physiol-Gastr Liver Physiol 2003; 284: G290-301.

33. Gagandeep S, Rajvanshi P, Sokhi RP, Slehria S, Palestro CJ, Bhargava KK, et al. Transplanted hepatocytes engraft, survive and proliferate in the liver of rats with carbon tetrachloride-induced cirrhosis. J Pathol 2000; 191: 78-85.

34. Cai J, Ito M, Nagata H, Westernman KA, Lafleur D, Chowdhury JR, et al. Treatment of liver failure in rats with end-stage cirrhosis by transplantation of immortalized hepatocytes. Hepatology 2002; 36: 386-94.

35. Tsukamoto H, Matsuoka M, French SW. Experimental models of hepatic fibrosis: a review. Semin Liver Dis 1990; 10: 56-65.

36. Yuan L-P, Chen FH, Ling L, Dou PF, Bo H, Zhong MM, Xia LJ. Protective effects of total flavonoids of Bidens pilosa L. (TFB) on animal liver injury and liver fibrosis. J Ethnopharmacol 2009; 116: 539-46. 